\title{
The neuropsychology of emerging psychosis and the role of working memory in episodic memory encoding
}

This article was published in the following Dove Press journal:

Psychology Research and Behavior Management

\author{
Marlon O Pflueger \\ Pasquale Calabrese ${ }^{2}$ \\ Erich Studerus ${ }^{3}$ \\ Ronan Zimmermann ${ }^{4}$ \\ Ute Gschwandtner ${ }^{4}$ \\ Stefan Borgwardt ${ }^{5}$ \\ Jacqueline Aston ${ }^{3}$ \\ Rolf-Dieter Stieglitz ${ }^{6}$ \\ Anita Riecher-Rössler ${ }^{3}$ \\ 'Department of Forensic Psychiatry, \\ University of Basel Psychiatric \\ Clinics, Basel, Switzerland; ${ }^{2}$ Division \\ of Molecular and Cognitive \\ Neuroscience, University of Basel, \\ Basel, Switzerland; ${ }^{3}$ Center for \\ Gender Research and Early Detection, \\ University of Basel Psychiatric \\ Hospital, Basel, Switzerland; \\ ${ }^{4}$ Department of Neurology and \\ Neurosurgery, Hospital of the \\ University of Basel, Basel, Switzerland; \\ ${ }^{5}$ Department of Psychiatry (UPK), \\ University of Basel, Basel, Switzerland; \\ ${ }^{6}$ Division of Clinical Psychology and \\ Psychiatry, University of Basel, Basel, \\ Switzerland
}

Correspondence: Marlon O Pflueger Department of Forensic Psychiatry, University of Basel Psychiatric Clinics, Wilhelm Klein-Str. 27, CH-40I2 Basel, Switzerland

Tel +4I 6 I 3255270

Email marlon.pflueger@upkbs.ch
Background: Episodic memory encoding and working memory (WM) deficits are among the first cognitive signs and symptoms in the course of schizophrenia spectrum disorders. However, it is not clear whether the deficit pattern is generalized or specific in nature. We hypothesized that encoding deficits at an early stage of the disease might be due to the more fundamental WM deficits. Methods: We examined episodic memory encoding and WM by administering the California Verbal Learning Test, a 2-back task, and the Wisconsin Card Sorting Test in 90 first-episode psychosis (FE) patients and 116 individuals with an at-risk mental state for psychosis (ARMS) compared to 57 healthy subjects.

Results: Learning progress, but not span of apprehension, was diminished to a similar extent in both the ARMS and the FE. We showed that this was due to WM impairment by applying a structural equation approach.

Conclusion: Thus, we conclude that verbal memory encoding deficits are secondary to primary WM impairment in emerging psychosis.

Keywords: at-risk mental state, first-episode psychosis, cognition, serial position effect, recency, semantic cluster ratio, 2-back task, rate of learning

\section{Introduction}

Current treatment strategies for schizophrenia aim at developing drugs specifically designed to enhance cognition and/or adopting an early detection and intervention approach in order to minimize symptom load and preserve functional capabilities. ${ }^{1-3}$

Both strategies depend on an accurate specification and a detailed description of the structure of cognitive deficits in the prodromal phase of the disease ${ }^{4}$ to provide either valid and reliable outcome measures for clinical trials or predictors for early detection approaches.

Structural and functional brain abnormalities are evident as early as in the prodromal phase of the disease. ${ }^{5,6}$ The same is true with cognitive deficits, where evidence indicates that the performance of patients in a prodromal phase of the disease lies between that of healthy controls and inferior performing patients in a first episode of psychosis. ${ }^{7-10}$

It is still debated, however, whether subjects at clinical high risk for psychosis or, interchangeably, with an at-risk mental state (ARMS), as well as first-episode psychosis (FE) patients, might display a deficit pattern of a specific or generalized nature, ie, whether a specifically circumscribed core deficit might explain the whole observable deficit pattern or not. ${ }^{11-14}$

It has been repeatedly reported that, among other domains, both working memory (WM) and verbal episodic memory are impaired in ARMS and FE patients. ${ }^{7,814-16}$ 
Unlike other cognitive domains affected, WM impairment is suggested to constitute a core feature of psychotic disorders. ${ }^{17-19}$ And because of its intermediate role between genotype and phenotype, it is generally regarded as an endophenotype in schizophrenia spectrum disorders. ${ }^{20,21}$ Even if the endophenotype concept has not been without criticism, ${ }^{22}$ it once again emphasizes the significance of WM impairment in schizophrenia spectrum disorders.

Impairment in verbal episodic memory has been interpreted in terms of an encoding deficit. ${ }^{23}$

In the present study, we adopted an approach which parsed the verbal memory encoding processes into smaller units, in order to shed some light on the kind of interdependence between both the episodic memory encoding deficit and the WM deficit in ARMS and FE patients. This is in line with recommendations regarding evidence on specific vulnerability markers for the prediction of psychosis. ${ }^{11}$

\section{Objectives}

The current study sought to elucidate the influence of WM on verbal episodic memory encoding by means of the serial position effect of free recall ${ }^{24}$ and the semantic cluster ratio (SCR) in ARMS and FE individuals. The SCR might reflect to what extent a subject engages in deep semantic encoding, ${ }^{25}$ which is much more demanding on WM than the shallow encoding of physical properties (eg, small/large letters, male/ female voice, etc.).

Serial position effects express nonuniform recall probabilities of memory items as a function of list position. The recency (rec) recall probability from the serial position curve is used as a surrogate measure of WM capacity ${ }^{24,26-28}$ and is related to learning curves derived from the California Verbal Learning Test (CVLT). ${ }^{29}$

Specific hypotheses are:

1. Compared to healthy control (HC) subjects, FE and ARMS individuals show an impaired encoding, as reflected by a reduced rate of learning and span of apprehension (recall performance regarding the first encoding trial).

2. Since WM performance in ARMS and FE patients is known to be impaired, we hypothesize that the rec effect of free recall (WM capacity component) and the SCR (central executive component of WM) is attenuated.

3. Moreover, we hypothesize a decreased impairment in episodic memory encoding after adjustment for WM deficiency. For that purpose, we additionally refer to explicit measures of WM, ie, response latency and omission errors from the N-back task and concept failures from the Wisconsin Card Sorting Test (WCST).

\section{Methods}

\section{Participants}

The study sample comprised $90 \mathrm{FE}$ and 116 ARMS subjects (Supplementary materials) who completed a neuropsychological assessment at baseline. All participants were recruited between March 2000 and May 2015 from the specialized Early Detection of Psychosis (FEPSY) Clinic, which is an Outpatient Department of the University of Basel Psychiatric Hospital, Basel, Switzerland (Riecher-Rössler et al, ${ }^{7,30}$ for details of study design and screening procedure). The severity of psychopathology was assessed using the Brief Psychiatric Rating Scale ${ }^{31}$ and the Scale for the Assessment of Negative Symptoms. ${ }^{32}$ Moreover, four selected Brief Psychiatric Rating Scale items served as decision criteria for the presence of manifest psychosis in the FEPSY project. Drug use was assessed with the Basel lnterview for Psychosis. ${ }^{33}$

In total, $57 \mathrm{HCs}$ were recruited by approaching students of a trade school, from hospital staff, and through advertisements. These subjects had no current psychiatric disorder, as assessed by a clinical interview, and no history of psychiatric illness, head trauma, neurological disease, serious medical or surgical illness, substance abuse, or family history of psychiatric disorders.

Exclusion criteria for patients were age younger than 18 years, insufficient knowledge of German language, IQ $<70$, previous episode of schizophrenic psychosis (treated with antipsychotics for $>3$ weeks), psychosis due to organic reasons or substance abuse, or psychotic symptoms within a clearly diagnosed affective psychosis or borderline personality disorder. The local ethics committee of the University of Basel approved all aspects of the study, and written informed consent was obtained from each participant.

\section{Memory assessment}

Episodic memory in ARMS, FE, and HC was assessed with a German version of the CVLT. ${ }^{29}$ This assessment requires immediate recall of an auditory presentation of a 16-item word list, made up of four semantic categories (drinks, clothes, fruits, and tools), across five trials.

The following variables were extracted: number of words correctly recalled during trial one through five and the SCR, defined as the number of consecutive recalls of word pairs in a same-category list, divided by what would be randomly expected, if the participant would not have used a categoryrelated recall strategy. Thus, an SCR of 1 reflects a semantic clustering completely at random, while an SCR $>1$ reflects a semantic clustering above random, ie, the adoption of a memory organization strategy based on the central executive component of the WM. 
Encoding efficiency was derived by both the intercept (span of apprehension) and the learning curves' slope, as function of the learning trials. The recall probabilities were computed item by item across the five trials. Each individual's serial position curve was represented as a series of four averaged recall probabilities (primacy, middle 1, middle 2, and rec - Supplementary materials).

WM was assessed with the Test Battery for Attentional Performance (TAP) WM task ${ }^{34}$ and a computerized German version of the WCST. ${ }^{35}$ The TAP WM task is a 2-back task requiring the participant to indicate whether a given stimulus (single-digit number appearing on the computer screen) matches a predecessor, two trials back. Task performance is reflected by number of omission errors and reaction time during correct responses.

\section{Statistical analysis}

Statistical and numerical analyses were performed using the R environment for statistical computing. ${ }^{36}$ In order to test for simple group differences, analysis of variance (ANOVA) or Kruskal-Wallis rank sum test was adopted. Frequency balance across two or more groups was inspected with Fisher's exact or $\chi^{2}$ tests. Missing values were subjected to imputation, such that the variance-covariance matrix of the data set remained unchanged (Supplementary materials). All subsequent analyses were performed based on the imputed data.

Learning performance of the CVLT was estimated using a log-linear limited growth mixed-effect model (Supplementary materials) that included an intrasubject random slope (for trial) and intercept. An ANOVA was performed on the linear mixed-effect model, based on marginal sums of squares (Type III Sum of Squares). Whenever necessary, log and trigonometric transforms were applied (Supplementary materials).

A structural equation modeling (using the package Lavaan 0.5-23.1097) approach, using maximum-likelihood estimation, was conducted in order to determine whether relevant factors, such as the TAP 2-back task, WCST concept failures, SCR, and rec effect, might contribute to word list encoding. Goodness of fit was assessed by calculating the root mean square error of approximation (RMSEA), where values of RMSEA $<0.08$ indicate appropriate fit. We, additionally, provided the comparative fit index (where $>0.93$ is generally considered a good fit) and the standardized root mean square residual $(<0.08)$.

Wherever multiple comparisons of mean were required, a multivariate ANOVA was performed, followed by multiple univariate ANOVA. Finally, Tukey's honest significant difference was computed.

\section{Results \\ Demographic characteristics and descriptive statistics}

The groups did not differ with respect to gender and education (Table 1). Unlike with cannabis use, the analyses of medication influences were limited to FE and ARMS, since $\mathrm{HC}$ did not use drugs. A significant proportion of FE (35.6\%), but only $6(5.2 \%)$ ARMS subjects, were treated with antipsychotics for less than 3 weeks at a maximum dose of below $125 \mathrm{mg}$ chlorpromazine equivalent per day. ARMS patients received significantly more frequently antidepressants as compared to FE. Finally, there was a significant difference with regard to cannabis use. The highest proportion of cannabis users was observed in FE patients (34\%), followed by ARMS patients $(27 \%)$, and, finally, HC ( $8 \%)$. The most intense use was likewise observed in FE (Table 1).

FE and ARMS patients did not differ in negative symptoms, but general psychopathology was somewhat more pronounced in $\mathrm{FE}$ as compared to ARMS patients $(\mathrm{U}=2076.5$, $p<0.001)$.

Table 2 shows aggregated raw memory and WCST scores of ARMS, FE, and HC. Except for SCR, HC always outperformed ARMS and FE significantly. There were no statistical effects of age, gender, education, or of any medical compounds listed in Table 2 on the WM performance measures.

\section{Encoding in word list learning}

A log-linear limited growth mixed-effect model was fit in order to estimate the recall-by-trial function and by taking into account trial and subject as random factors. To model the inflating intertrial variance, a power variance function was additionally fit, using trial as covariate. For confounder selection, a sequential variable introduction and step-down approach was adopted, considering medication, cannabis use, and demographic variables as potential confounders.

An ANOVA indicated a main effect of $\log ($ trial) $(\mathrm{F}(1,1048)=2275.9 ; p<0.001)$ and a significant interaction $\log ($ trial $) \times \operatorname{group}(\mathrm{F}(2,1048)=10.1 ; p<0.001)$, but no main effect of group $(\mathrm{F}(2,258)=1.5 ; p=0.216)$. Additionally, we found a main effect of tranquillizer $(\mathrm{F}(1,258)=5.2 ; p=0.023)$ and $\operatorname{sex}(\mathrm{F}(1,258)=13.3 ; p<0.001)$, and an interaction $\log ($ trial $) \times$ cannabis use $(\mathrm{F}(1,1048)=6.4 ; p=0.012)$, with cannabis use coded dichotomously, frequent (several times per week or daily) or less frequent use. 
Table I Demographic characteristics and psychopathology of ARMS subjects, FE patients, and HC

\begin{tabular}{|c|c|c|c|c|}
\hline Variables & ARMS (N=I16) & FE $(\mathbf{N}=90)$ & $\mathrm{HC}(\mathrm{N}=57)$ & Statistics \\
\hline Age, years, M (SD) & $25.7(6.5)$ & $28.3(7.9)$ & $24.9(6.4)$ & $\chi^{2}=154.1(148), p=0.349^{a}$ \\
\hline Sex (female), N (\%) & $36(31)$ & $33(37)$ & $26(46)$ & $\chi^{2}=3.5(2), p=\left.0.17\right|^{b}$ \\
\hline \multicolumn{5}{|l|}{ Education, N (\%) } \\
\hline$<9$ years & $13(11.2)$ & $14(15.6)$ & $2(3.5)$ & \multirow[t]{4}{*}{$\chi^{2}=8.2(6), p=0.224^{b}$} \\
\hline $9-11$ years & $36(3 I)$ & $33(36.7)$ & $17(29.8)$ & \\
\hline $12-13$ years & $4 \mathrm{I}(35.3)$ & $27(30)$ & $26(45.6)$ & \\
\hline 14-20 years & $26(22.4)$ & $16(17.8)$ & $12(21.1)$ & \\
\hline \multicolumn{5}{|l|}{ Medication, N (\%) } \\
\hline Neuroleptics & $6(5.2)$ & $32(35.6)$ & 0 & $p<0.00 I^{c}$ \\
\hline Antidepressants & $39(33.6)$ & $17(18.9)$ & 0 & $p=0.027^{c}$ \\
\hline Tranquilizers & $20(17.2)$ & $19(21.1)$ & 0 & $p=0.59 \mathrm{I}^{\mathrm{c}}$ \\
\hline \multicolumn{5}{|l|}{ Cannabis, N (\%) } \\
\hline No & $81(73.0)$ & $54(65.9)$ & $12(92.3)$ & \multirow[t]{6}{*}{$\chi^{2}=7.3(8), p=0.508^{b}$} \\
\hline Less than monthly & $7(6.3)$ & $6(7.3)$ & I (7.7) & \\
\hline Monthly & $4(3.6)$ & $\mathrm{I}(\mathrm{I} .2)$ & $0(0)$ & \\
\hline Weekly & $10(9.0)$ & $10(12.2)$ & $0(0)$ & \\
\hline Daily & $9(8.1)$ & II (I3.4) & $0(0)$ & \\
\hline Data not available & 5 & 8 & 44 & \\
\hline BPRS global score, M (SD) & $40.4(9.3)$ & $52.8(11.9)$ & 0 & $U=2076.5, p<0.00 I^{d}$ \\
\hline SANS summary score, $M(\mathrm{SD})$ & $7.2(4.8)$ & $7(5)$ & 0 & $\mathrm{U}=5375, p=0.715^{d}$ \\
\hline
\end{tabular}

Notes: 'aruskal-Wallis Rank Sum Test for more than two samples. ${ }^{b}$ Pearson $\chi^{2}$ Tests. 'Fisher's Exact Test (only ARMS vs FE). ${ }^{d}$ Mann-Whitney Rank Sum Test for two samples (only ARMS vs FE). "-" = no data.

Abbreviations: ARMS, at-risk mental state for psychosis; BPRS, Brief Psychiatric Rating Scale; FE, first-episode psychosis; HC, healthy control; SANS, Scale for the Assessment of Negative Symptoms; M, mean, SD, standard deviation.

Table 2 Mean, SD, and MD of the neuropsychological variables (raw values)

\begin{tabular}{|c|c|c|c|c|c|c|c|c|c|c|}
\hline \multirow{2}{*}{$\begin{array}{l}\text { Neuropsychological } \\
\text { variables }\end{array}$} & \multicolumn{3}{|c|}{ ARMS } & \multicolumn{3}{|l|}{ FE } & \multicolumn{3}{|l|}{$\mathrm{HC}$} & \multirow[t]{2}{*}{ Statistics $^{\mathbf{a}}$} \\
\hline & $M$ & MD & SD & $M$ & MD & SD & $M$ & MD & SD & \\
\hline Trial I & 8 & 8 & 2.2 & 7.4 & 7 & 2.3 & 8.4 & 9 & 2.1 & $\chi^{2}=8.9(2), p=0.012^{b}$ \\
\hline Trial 5 & 14 & 14 & 1.8 & 12.9 & 14 & 2.6 & 14.7 & 15 & 1.3 & $\chi^{2}=23.9(2), p<0.00 I^{c}$ \\
\hline Tot. Recall & 59.2 & 60 & 9.1 & 54.8 & 55 & 10.6 & 62.8 & 64 & 7.4 & $\chi^{2}=24.0(2), p<0.00 I^{c}$ \\
\hline SCR & 1.7 & 1.6 & 0.9 & 1.6 & 1.4 & 0.7 & 2 & 1.9 & I & $\chi^{2}=4.3(2), p=0.116$ \\
\hline WM (RT) & 695.9 & 662.9 & 211 & 734.4 & 692.7 & 225.2 & 581.5 & 528.4 & 165.9 & $\chi^{2}=24.4(2), p<0.00 I^{c}$ \\
\hline WM (Mis) & 2.8 & 2 & 2.6 & 3.4 & 3 & 2.8 & 1.6 & I & 1.7 & $\chi^{2}=14.5(2), p=0.001$ \\
\hline WCST (cfail) & 0.8 & 0 & 1.1 & 1.3 & I & 1.5 & 0.6 & 0 & 1.0 & $\chi^{2}=11.6(2), p=0.003^{b}$ \\
\hline WCST (Pers) & 4.8 & 2 & 6.7 & 7.6 & 4 & 8.2 & 3.8 & I & 6.7 & $\chi^{2}=14.4(2), p=0.00 I^{b}$ \\
\hline
\end{tabular}

Notes: ${ }^{a}$ Kruskal-Wallis Rank Sum Test for more than two samples. Trial I, $5=$ Number correct recalled items during trial I, 5 respectively. Tot. Recall = Number of correctly recalled list items summed across all five trials. Post hoc comparisons indicated: bHC, ARMS $>$ FE, ' $\mathrm{HC}>\mathrm{ARMS}>\mathrm{FE}$, ${ }^{\mathrm{d}} \mathrm{HC}>\mathrm{ARMS}$, FE.

Abbreviations: ARMS, at-risk mental state for psychosis; FE, first-episode psychosis; HC, healthy control; M, mean; MD, median; SCR, semantic cluster ratio; SD, standard deviation; TAP, Test Battery for Attentional Performance; WCST (cfail), Wisconsin Card Sorting Test concept failure; WCST (Pers), Wisconsin Card Sorting Test perseverative errors; WM (RT), TAP 2-Back Task Response Time; WM (Mis), TAP 2-Back task number of omission errors.

Table 3 Estimated intercept and slope of the learning curves of female ARMS individuals, FE patients, and $\mathrm{HC}$ adjusted for tranquilizer and cannabis use

\begin{tabular}{lllll}
\hline Learning & ARMS & FE & HC & Statistics $^{\mathbf{a}}$ \\
\hline Growth-rate k & 0.79 & 0.67 & 0.92 & HC > ARMS, FE \\
Intercept $b_{0}$ & 9.15 & 8.63 & 9.18 & NS \\
\hline
\end{tabular}

Notes: Growth-rate $k=$ rate of learning. Intercept $b_{0}=$ Span of apprehension. ${ }^{a}$ Contrast analysis derived from the linear mixed-effect model (see text for statistical details).

Abbreviations: ARMS, at-risk mental state for psychosis; FE, first-episode psychosis; HC, healthy control; NS, not significant.
As the contrast analysis indicated, both ARMS $(t=2.5$; $d f=1,048 ; p=0.012)$ and FE $(t=4.5 ; d f=1,048 ; p<0.001)$ showed a smaller rate of learning (slope) than HC (Table 3 ). The rate of learning was also reduced by the use of cannabis $(t=2.5 ; d f=1048 ; p=0.012)$. Male gender was associated with a smaller intercept (span of apprehension) $(t=3.6 ; d f=258$; $p<0.001)$, as was the use of tranquillizers $(t=2.3 ; d f=258$; $p=0.023$. 


\section{The serial position effect of free recall}

Figure $1 \mathrm{~A}$ and $\mathrm{B}$ shows the recall probability of the memory items as a function of their serial position. Marked elevations at both ends indicate a strong primacy and a somewhat weaker rec effect. In contrast, the intermediate memory items yielded lower recall probabilities. However, in FE, there appears to be a region of exceptionally low recall probability at the level of mid2 (memory items 9-12).

Positional recall probabilities were arcsine transformed and modeled by a multivariate ANOVA. Group, gender, age, education, neuroleptics, antidepressants, and cannabis use were modeled as independent variables. The multivariate ANOVA indicated a highly significant group effect (Wilks- $\lambda=0.89 ; \mathrm{F}(8,512)=3.9 ; p<0.001$ ) and a trend for a gender effect (Wilks- $\lambda=0.96 ; \mathrm{F}(4,256)=2.4 ; p=0.052)$. Since none of the remaining variables showed a statistically significant relationship with the positional recall probabilities, they were excluded from further analyses.
A series of univariate ANOVAs indicated significant group differences at all serial positions, with effect sizes coarsely in increasing order $(\operatorname{prim}(\mathrm{F}(2,259)=3.3 ; p<0.040)$, mid1 $(\mathrm{F}(2,259)=6.4 ; p=0.002), \operatorname{mid} 2(\mathrm{~F}(2,259)=13.2 ; p<0.001)$, and rec $(\mathrm{F}(2,259)=7.4, p<0.001)$. Similarly, gender effects increased steadily from prim $(\mathrm{F}((1,259)=3.2, p=0.072)$, mid1 $(\mathrm{F}(1,259)=4.1, p=0.044), \operatorname{mid} 2(\mathrm{~F}(1,259)=4.5, p=0.035)$, to $\operatorname{rec}(\mathrm{F}(1,259)=7.0, p=0.009)$.

Post hoc honest significant difference indicated a significantly lower primacy recall probability in FE compared to HC ( $p=0.035)$. At the level of mid1 and mid2, both ARMS and $\mathrm{HC}$ performed significantly superior compared to FE. At the level of the rec region, $\mathrm{HC}$ showed a significantly higher recall probability compared to both the $\mathrm{FE}(p<0.001)$ and the ARMS $(p=0.031)$ patients. There was no significant difference between ARMS and FE patients. Thus, contrary to the pre-recency portion of the word list, the rec region exclusively showed an $\mathrm{HC}$ superiority effect.

A

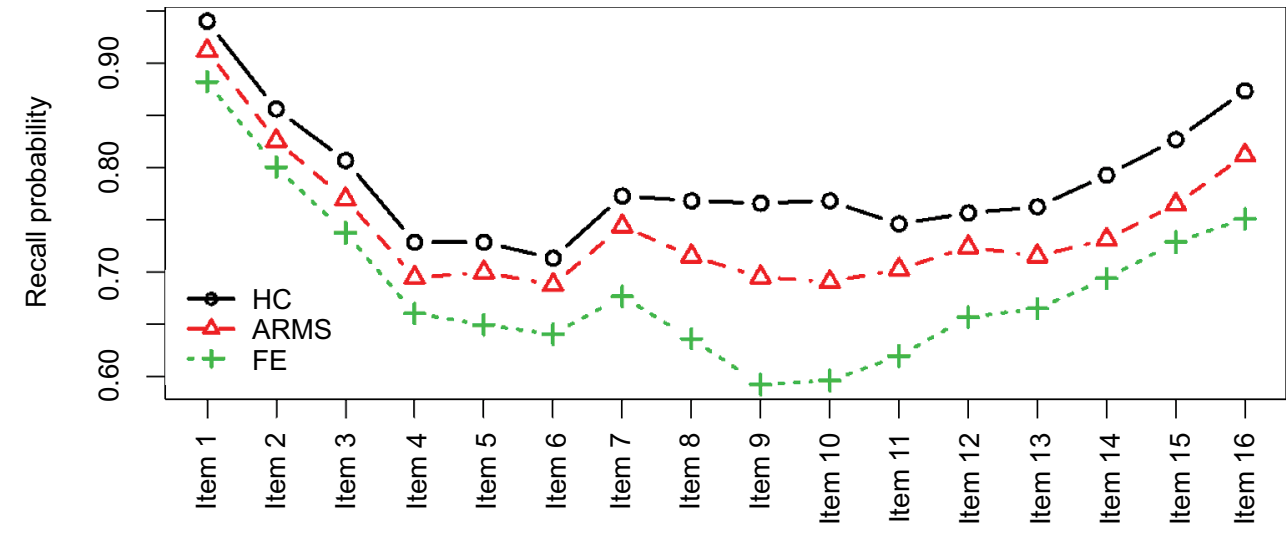

B

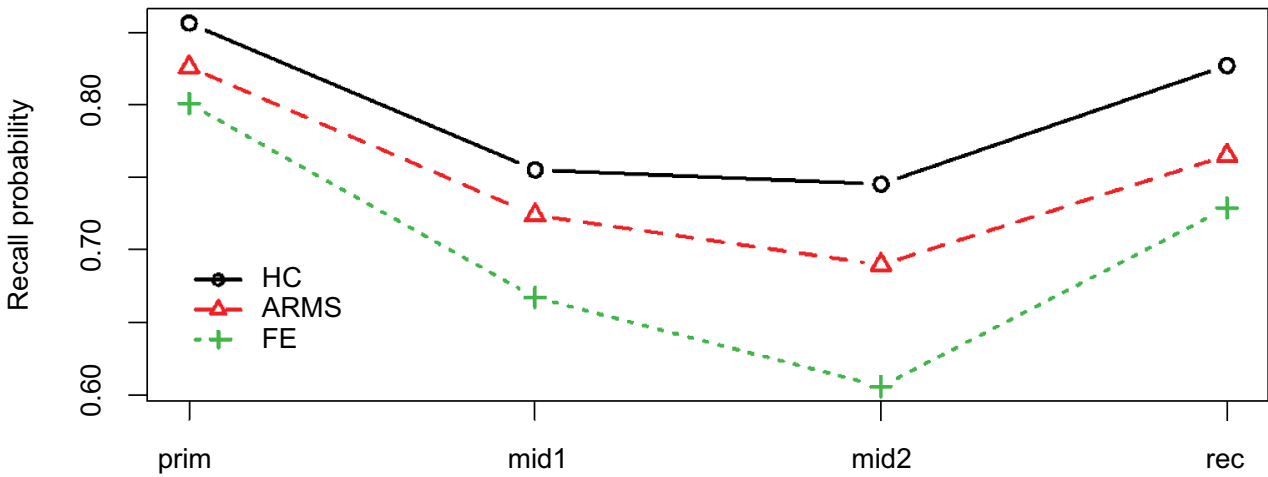

Figure I The serial position effects of the CVLT learning trials according to ARMS subjects, FE patients, and HC.

Notes: Recall probability of list items as function of each item's serial position (A) and the aggregated recall probability (B) according to the primacy region (prim - first quarter), middle I (mid I - second quarter), middle 2 (mid2 - third quarter), and the rec region (rec - last quarter) of ARMS subjects, FE patients, and HC. Recall probabilities at the level of mid2 are significantly lower in FE compared to ARMS subjects and $\mathrm{HC}$. rec recall probabilities differ significantly between $\mathrm{HC}$ and $\mathrm{FE}$ and at the level of a trend between $\mathrm{HC}$ and ARMS subjects (see text for statistical details).

Abbreviations: ARMS, at-risk mental state for psychosis; FE, first-episode psychosis; HC, healthy control; mid, middle; prim, primacy; rec, recency. 


\section{Compromised learning as result of WM deficit}

According to the hypothesis that a primary WM deficit accounts for deficits observed in verbal learning of FE and ARMS patients, we constructed a confirmatory structural equation model with an exogenous latent variable "WM". This latent variable was constructed from maintenance WM (rec effect) and the central executive component of WM (SCR and WCST concept failure). To assure construct validity, the TAP 2-back tasks omission errors and response latency were fixed to the latent variable as composite measure central executive component of the WM (WMc). Where appropriate, variables were adjusted for confounders and $z$-transformed (Supplementary materials).

In order to explain the learning disabilities in FE and ARMS patients, it was sufficient to explain the difference between the group's specific rates of learning (explained in

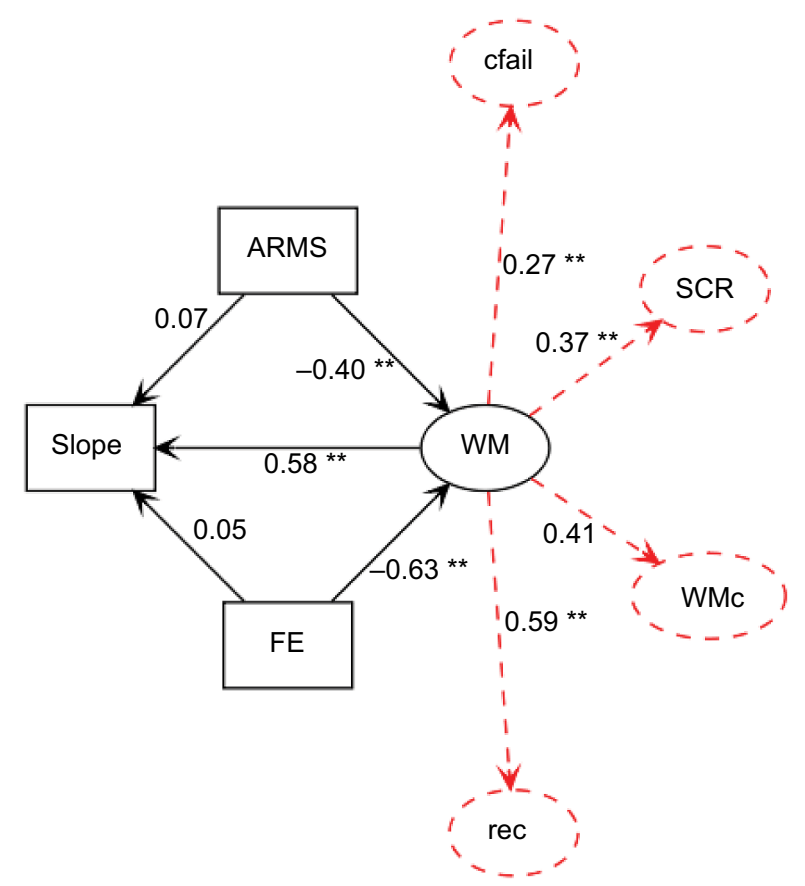

Figure 2 Relationship between ARMS subjects, FE patients, rate of learning (slope), and $W M$ as modeled by means of structural equations $(\mathrm{N}=263)$.

Notes: WM is an exogenous latent variable, ie, the common factor of rec, SCR, cfail, and WMc (composite of 2-back task performance), as indicated by dashed lines. All parameters are provided as standardized values. The WMc $(\gamma=0.4 \mathrm{I})$ was fixed to the WM, and the loadings of rec $(\gamma=0.59 ; z=4.5 ; p<0.001), \operatorname{SCR}(\gamma=0.37 ; z=3.7$; $p<0.00 \mathrm{I})$, and WCST concept failure $(\gamma=0.27 ; z=3.0 ; p=0.003)$ were allowed to vary freely. WM moderately predicted rate of learning $(\gamma=0.58 ; z=3.7 ; p<0.001)$. The ARMS $(\gamma=-0.40 ; z=-3.1 ; p=0.002)$ and the FE patients $(\gamma=-0.63 ; z=-4.2 ; p<0.001)$ had significantly different means on WM compared to $\mathrm{HC}$, but are not anymore associated with the learning rate. Thus, impaired WM explains encoding deficits in verbal learning $(* * p \leq 0.001)$.

Abbreviations: ARMS, at-risk mental state for psychosis; FE, first-episode psychosis; HC, healthy control; rec, recency; SCR, semantic cluster ratio; cfail, Wisconsin Card Sorting Test concept failure; WM, working memory; WMc, central executive component of the WM. the "Encoding in word list learning" section). Therefore, an individual regression-based rate of learning (slope) adjusted for cannabis use was determined according to each participant. The result is shown as a path diagram using standardized coefficients (Figure 2).

The $\chi^{2}$ test of model fit $\left(\chi^{2}(11)=18.0 ; p=0.081\right)$ suggested the model's appropriateness. This was further supported by its RMSEA $=0.049$ CI90 $(0.0,0.9)$, its CFI $=0.94$, and its $\mathrm{SRMR}=0.037$. As a result of the WM's mediator role, the direct association between both the FE ( $\gamma=0.05, z=0.5$; $p=0.649)$ and the ARMS patients $(\gamma=0.07, z=0.8 ; p=0.447)$, with regard to the rate of learning, was no longer statistically significant.

\section{Discussion}

We provide evidence that verbal learning is moderately compromised in FE and ARMS patients. The same held true with regard to WM performance in explicit tests of WM (n-back, WCST). However, the impairment in episodic memory encoding affected only the rate of learning and did not influence the span of apprehension (Hypothesis I). As the analysis of the serial position recall probabilities revealed, for both FE and ARMS patients, the source of this reduced performance was mainly traced back to the rec proportion of the word list (Hypothesis II), while in FE patients it was additionally due to the pre-recency proportion. Apart from gender, the rate of learning was not dependent on potential confounders like age, medication, or cannabis use. ${ }^{37}$ Thus, the rec region was the only source for the reduced rate of learning in ARMS patients.

According to the view that the rec effect reflects maintenance $\mathrm{WM},{ }^{24,26-28}$ we provided additional evidence for a compromised WM in FE and ARMS patients. The dysfunctional short-term memory storage might have been especially important in explaining a diminished encoding efficiency of list items into episodic memory.

In order to test this, we formulated a hypothesis in terms of a structural equation model. In support of our hypothesis, the latent exogenous variable WM was significantly and positively related to the rate of learning. Most importantly, the FE and ARMS patients showed worse WM performance than HC. However, the rate of learning was no longer statistically different between groups when corrected for the influence of WM. Therefore, we conclude that WM might essentially contribute to the deficits in the formation of a long-term episodic memory in both the FE and the ARMS patients (Hypothesis III).

However, an issue arises from theoretical considerations, and refers to the nature of the rec effect itself. There 
is a controversial discussion in psychological literature on whether the rec effect in verbal learning truly reflects WM capacity. Initially, this idea emerged from experiments that demonstrated dissociable effects on rec and pre-recency portions of word lists. ${ }^{38-40}$ However, the observation of a long-term rec effect in so-called continuous distractor free recall tasks posed a challenge to this point of view, ${ }^{41}$ since these tasks are characterized by presenting memory items along with distracting tasks, prior to and after each item, such that interference with WM should abolish the rec effect. On the other hand, both continuous distracter free recall rec and immediate free recall rec might be manipulated by experimental conditions, such that a double dissociation is evident. ${ }^{42}$ Thus, regarding this issue, an unequivocal conclusion cannot be drawn.

Overall, the evidence we reported grossly confirmed our hypotheses. However, the picture that emerged was not quite as uniform as the hypotheses implied. First, the impairment in episodic memory encoding spared the span of apprehension and was exclusively restricted to the rate of learning. Second, even though the N-back task indicated a reduced central executive component of WM in both ARMS and FE patients, the SCR was not different between the groups.

Evidence of an aberrant rec effect in schizophrenia is sparse and inconsistent. Although there are reports on a diminished rec effect in schizophrenia, ${ }^{43,44}$ some found an elevated rec effect compared to $\mathrm{HC},{ }^{45}$ and yet some others found no difference at all. ${ }^{23,46,47}$ And, finally, there is virtually no evidence regarding ARMS patients. Therefore, our unequivocal and clear-cut findings of a reduced rec recall probability in both FE and ARMS patients might add to a more concise picture of the cognitive deficit in these groups.

We not only provided evidence that the maintenance (rec effect) but also the central executive component of the WM was impaired in both ARMS and FE patients. A general impairment of WM has been demonstrated in both patients with fully developed schizophrenia ${ }^{48,49}$ and those experiencing a first episode of psychosis. ${ }^{4}$ But it also occurs in prodromal patients (ie, ARMS patients with later transition), and ARMS patients in general. ${ }^{7,815,50}$ However, SCR was preserved, which is somewhat puzzling, given the general importance of the WM in semantic encoding ${ }^{51,52}$ and the large body of evidence indicating a reduced tendency in using semantic cues as to effectively improve memory performance in schizophrenia. ${ }^{53-58}$

The results from the structural equation model suggested that the impaired WM is sufficient in explaining a reduced rate of learning in ARMS and FE patients. Support for this finding is suggested by evidence emphasizing the role of WM for episodic memory encoding in schizophrenia. $59,46,60,56,58,61$ Thus, verbal memory encoding deficits are secondary to a primary WM impairment and, therefore, are contrary to assumptions of a generalized-deficit perspective. ${ }^{11-14}$

However, it appears that additional factors could have contributed to the observed encoding deficiencies in FE patients, as their particular low performance in pre-recency portions suggested.

This last issue surely warrants replication and a finer grained analysis. There is evidence that long-term memory encoding and the central executive component of the WM share considerable proportions of prefrontal resources and that the recruitment of these prefrontal areas is less behavioral efficient in patients with schizophrenia as compared to HC subjects. ${ }^{60,61}$ It remains to be shown whether and how this mechanism gradually applies to ARMS and FE patients and might, thus, correlate with a diminishing encoding efficiency in emerging psychosis.

\section{Acknowledgment}

The data on which this research is based were drawn from the FEPSY (Early Detection) Study, which is funded by project grants from the Swiss National Science Foundation (Nos. 3200-057216-99; 3200-057216/3). We thank all patients and volunteers who participated in the study as well as the referring specialists. We especially would like to thank Dr Christian Schindler for his support with statistical advices.

\section{Disclosure}

The authors report no conflicts of interest in this work.

\section{References}

1. Nordentoft M, Melau M, Iversen T, et al. From research to practice: how OPUS treatment was accepted and implemented throughout Denmark. Early Interv Psychiatry. 2015;9(2):156-162.

2. Buchanan RW, Davis M, Goff D, et al. A summary of the FDA-NIMHMATRICS workshop on clinical trial design for neurocognitive drugs for schizophrenia. Schizophr Bull. 2005;31(1):5-19.

3. Insel TR. Rethinking schizophrenia. Nature. 2010;468(7321):187-193.

4. Mesholam-Gately RI, Giuliano AJ, Goff KP, Faraone SV, Seidman LJ. Neurocognition in first-episode schizophrenia: a meta-analytic review. Neuropsychology. 2009;23(3):315-336.

5. Smieskova R, Fusar-Poli P, Allen P, et al. Neuroimaging predictors of transition to psychosis - A systematic review and meta-analysis. Neurosci Biobehav Rev. 2010;34(8):1207-1222.

6. Fusar-Poli P, Broome MR, Matthiasson P, et al. Spatial working memory in individuals at high risk for psychosis: longitudinal fMRI study. Schizophr Res. 2010;123(1):45-52.

7. Riecher-Rössler A, Pflueger MO, Aston J, et al. Efficacy of using cognitive status in predicting psychosis: a 7-year follow-up. Biol Psychiatr. In press 2009.

8. Fusar-Poli P, Deste G, Smieskova R, et al. Cognitive functioning in prodromal psychosis: a meta-analysis. Arch Gen Psychiatry. 2012;69(6):562-571. 
9. Studerus E, Papmeyer M, Riecher-Rössler A. Neurocognition and Motor Functioning in the Prediction of Psychosis. Vol 181. Karger Publishers; 2016. Available from: http://www.karger.com/Article/Abstract/440919. Accessed February 19, 2016.

10. Hauser M, Zhang JP, Sheridan EM, et al. Neuropsychological test performance to enhance identification of subjects at clinical high risk for psychosis and be most promising for predictive algorithms for conversion to psychosis: a meta-analysis. J Clin Psychiatr. 2017;78(01):e28-e40.

11. Brewer WJ, Wood SJ, Phillips LJ, et al. Generalized and specific cognitive performance in clinical high-risk cohorts: a review highlighting potential vulnerability markers for psychosis. Schizophr Bull. 2006;32(3):538-555.

12. Lencz T, Smith CW, McLaughlin D, et al. Generalized and specific neurocognitive deficits in prodromal schizophrenia. Biol Psychiatr. 2006;59(9):863-871.

13. Pukrop R, Schultze-Lutter F, Ruhrmann S, et al. Neurocognitive functioning in subjects at risk for a first episode of psychosis compared with first-and multiple-episode schizophrenia. J Clin Exp Neuropsychol. 2006;28(8):1388-1407.

14. Seidman LJ, Giuliano AJ, Meyer EC, et al. Neuropsychology of the prodrome to psychosis in the NAPLS consortium: relationship to family history and conversion to psychosis. Arch Gen Psychiatry. 2010;67(6):578-588.

15. Pflueger MO, Gschwandtner U, Stieglitz RD, Riecher-Rössler A. Neuropsychological deficits in individuals with an at risk mental state for psychosis - working memory as a potential trait marker. Schizophr Res. 2007;97(1-3):14-24.

16. Bora E, Murray RM. Meta-analysis of cognitive deficits in ultrahigh risk to psychosis and first-episode psychosis: do the cognitive deficits progress over, or after, the onset of psychosis? Schizophr Bull. 2014;40(4):744-755.

17. Castner S, Goldman-Rakic P, Williams G. Animal models of working memory: insights for targeting cognitive dysfunction in schizophrenia. Psychopharmacology. 2004;174(1).

18. Elvevag B, Goldberg TE. Cognitive impairment in schizophrenia is the core of the disorder. Crit Rev Neurobiol. 2000;14(1):1-21.

19. Gold JM, Carpenter C, Randolph C, Goldberg TE, Weinberger DR. Auditory working memory and Wisconsin Card Sorting Test performance in schizophrenia. Arch Gen Psychiatry. 1997;54(2):159.

20. Gottesman II, Gould TD. The endophenotype concept in psychiatry: etymology and strategic intentions. Am J Psychiatry. 2003;160(4):636-645.

21. Park S, Gooding DC. Working memory impairment as an endophenotypic marker of a schizophrenia diathesis. Schizophr Res Cogn. 2014;1(3):127-136.

22. Flint J, Munafò MR. The endophenotype concept in psychiatric genetics. Psychol Med. 2007;37(02):163.

23. Hill KS, Beers SR, Kmiec JA, Keshavan MS, Sweeney JA. Impairment of verbal memory and learning in antipsychotic-naïve patients with first-episode schizophrenia. Schizophr Res. 2004;68(2-3):127-136.

24. Murdock Jr BB. The serial position effect of free recall. J Exp Psychol. 1962;64(5):482-488.

25. Craik F, Lockhart R. Levels of processing: a framework for memory research. J Verbal Learn Verbal Behav. 1972;11(6):671-684.

26. Anderson JR, Bothell D, Lebiere C, Matessa M. An integrated theory of list memory. J Memory Lang. 1998;38(4):341-380.

27. Glanzer M. Storage Mechanisms in Recall. In: Bower GH, editor. The Psychology of Learning and Motivation: Advances in Research and Theory. Vol 5. Academic Press; 1972:129-193. Available from: http:// www.sciencedirect.com/science/article/B7J17-4S81B93-6/2/50dac4b 99510365ee1a36d3afc8c655d. Accessed June 15, 2010.

28. Waugh NC, Norman DA. Primary memory. Psychol Rev. 1965;72(2):89-104.

29. Delis D, Kramer J, Kaplan E, Ober B. California verbal learning test. Test Critiq. 1984:158.

30. Riecher-Rössler A, Gschwandtner U, Aston J, et al. The Basel earlydetection-of-psychosis (FEPSY)-study design and preliminary results. Acta Psychiatrica Scandinavica. 2007;115:114-125.

31. Lukoff D, Nuechterlein KH, Ventura J. Manual for the expanded brief psychiatric rating scale. Schizophr Bull. 1986;12:594-602.
32. Andreasen NC. The Scale for the Assessment of Negative Symptoms (SANS): conceptual and theoretical foundations. BrJ Psychiatry Suppl. 1989;(7):49-58

33. Riecher-Rössler A, Ackermann T, Uttinger M, et al. Das Basler Interview für Psychosen (BIP): Struktur, Reliabilität und Validität. Fortschritte der Neurologie · Psychiatrie. 2015;83(02):99-108.

34. Zimmermann P, Fimm B. Test for attentional performance (TAP). Herzogenrath, Germany: PsyTest. 1995.

35. Drühe-Wienholt CM, Wienholt W. CKV: Computergestütztes Kartensortierverfahren. Frankfurt am Main: Swets und Zeitlinger Testservices. 1998.

36. R Development Core Team. R: A Language and Environment for Statistical Computing. Vienna, Austria: R Foundation for Statistical Computing; 2015. Available from: http://www.R-project.org.

37. Aleman A, Hijman R, de Haan EHF, Kahn RS. Memory impairment in schizophrenia: a meta-analysis. Am J Psychiatry. 1999;156(9):1358-1366.

38. Glanzer M, Cunitz AR. Two storage mechanisms in free recall. JVerbal Learn Verbal Behav. 1966;5(4):351-360.

39. Postman L, Phillips LW. Short-term temporal changes in free recall. Quarter J Exp Psychol. 1965;17(2):132-138.

40. Unsworth N, Engle RW. The nature of individual differences in working memory capacity: active maintenance in primary memory and controlled search from secondary memory. Psychol Rev. 2007;114(1):104-132.

41. Bjork RA, Whitten WB. Recency-sensitive retrieval processes in longterm free recall. Cognit Psychol. 1974;6(2):173-189.

42. Davelaar E, Goshen-Gottstein Y, Ashkenazi A, Haarmann H, Usher M. The demise of short-term memory revisited: Empirical and computational investigations of recency effects. Psychol Rev. 2005;112(1):3-42.

43. Landrø NI, Ørbeck AL, Rund BR. Memory functioning in chronic and non-chronic schizophrenics, affectively disturbed patients and normal controls. Schizophr Res. 1993;10(1):85-92.

44. Stirling JD, Hellewell JSE, Hewitt J. Verbal memory impairment in schizophrenia: no sparing of short-term recall. Schizophr Res. 1997;25(2):85-95.

45. Egeland J, Sundet K, Rund BR, et al. Sensitivity and specificity of memory dysfunction in schizophrenia: a comparison with major depression. J Clin Exp Neuropsychol. 2003;25(1):79.

46. Chan AS, Kwok IC, Chiu H, Lam L, Pang A, Chow L. Memory and organizational strategies in chronic and acute schizophrenic patients. Schizophr Res. 2000;41(3):431-445.

47. Peuskens J, de Hert M, Janssen F, Hulselmans J, D'Haenens M, Sabbe B. Memory disorders in schizophrenia. In: De Deyn PP, Thiery E, D'Hooge R, editors. Memory: Basic Concepts, Disorders, and Treatment. 1st ed. Leuven: ACCO; 2003:488.

48. Forbes NF, Carrick LA, McIntosh AM, Lawrie SM. Working memory in schizophrenia: a meta-analysis. Psychol Med. 2009;39(06):889-905.

49. Kim J, Glahn DC, Nuechterlein KH, Cannon TD. Maintenance and manipulation of information in schizophrenia: further evidence for impairment in the central executive component of working memory. Schizophr Res. 2004;68(2-3):173-187.

50. Eastvold AD, Heaton RK, Cadenhead KS. Neurocognitive deficits in the (putative) prodrome and first episode of psychosis. Schizophr Res. 2007;93(1-3):266-277.

51. Demb JB, Glover H. Semantic encoding. J Neurosci. 1995;15(9): 5870-5878.

52. Wagner AD, Paré-Blagoev EJ, Clark J, Poldrack RA. Recovering meaning: left prefrontal cortex guides controlled semantic retrieval. Neuron. 2001;31(2):329-338

53. Bonner-Jackson A, Yodkovik N, Csernansky JG, Barch DM. Episodic memory in schizophrenia: the influence of strategy use on behavior and brain activation. Psychiatr Res. 2008;164(1):1-15.

54. Bonner-Jackson A, Haut K, Csernansky JG, Barch DM. The influence of encoding strategy on episodic memory and cortical activity in schizophrenia. Biol Psychiatr. 2005;58(1):47-55.

55. Wolf DH, Gur RC, Valdez JN, et al. Alterations of fronto-temporal connectivity during word encoding in schizophrenia. Psychiatr Res. 2007;154(3):221-232. 
56. Brébion G, David AS, Jones H, Pilowsky LS. Semantic organization and verbal memory efficiency in patients with schizophrenia. Neuropsychology. 2004;18(2):378-383.

57. Ragland JD, Gur RC, Valdez JN, et al. Levels-of-processing effect on frontotemporal function in schizophrenia during word encoding and recognition. Am J Psychiatry. 2005;162(10):1840-1848.

58. Achim AM, Lepage M. Episodic memory-related activation in schizophrenia: meta-analysis. Br J Psychiatry. 2005;187(6): 500-509.
59. Brébion G, Amador X, Smith MJ, Gorman JM. Mechanisms underlying memory impairment in schizophrenia. Psychol Med. 1997;27(02):383-393.

60. Barch DM, Csernansky JG, Conturo T, Snyder AZ. Working and longterm memory deficits in schizophrenia: is there a common prefrontal mechanism? J Abnorm Psychol. 2002;111(3):478-494.

61. Ragland JD, Blumenfeld RS, Ramsay IS, et al. Neural correlates of relational and item-specific encoding during working and long-term memory in schizophrenia. NeuroImage. 2012;59(2):1719-1726. 


\section{Supplementary materials Estimation of learning curves}

The graph of the average numbers of correctly recalled list items as a function of consecutive trials usually resembles a bounded growth curve, which can be formalized as follows:

$$
\text { recall(trial) }=\mathrm{S}-\mathrm{ce}^{-\mathrm{klog}(\text { trial })}
$$

where $\mathrm{c}$ is a group-specific constant and $\mathrm{S}$ is the growth limit, which can be set to 16 , since no more than 16 words can be learned (actually, the growth limit was set to $S=17$ in order to avoid taking the logarithm of zero). Moreover, $\mathrm{S}-\mathrm{c}$ represents the intercept (span of apprehension, ie, the maximum number of words that can be correctly assessed after the first list presentation) and $\mathrm{k}$ the learning slope (growth rate). The larger $\mathrm{k}$ is, the more and faster learning occurs. It determines how fast the amount of recalled list items converges toward the growth boundary S. Actually, the analysis resulted in a model with additional parameters, thus, extending the above formalization, as follows:

$$
\text { recall(trial) }=\mathrm{S}-\mathrm{ce}^{-\mathrm{klog}(\text { trial)(group+thc)+sex+trx }} .
$$

In expanding the expression, we obtain

$$
\text { recall(trial) }=\mathrm{S}-\mathrm{ce}^{\mathrm{sex}} \mathrm{e}^{\mathrm{t} t \mathrm{x}} \mathrm{e}^{-\mathrm{klog}(\text { trial)(grouptthc). }}
$$

Now the intercept becomes $\mathrm{S}-\mathrm{ce}^{\mathrm{sex}} \mathrm{e}^{\mathrm{ttx}}$,

where trx (tranquilizer use) and sex are dichotomously coded. It is important to note that the intercept is not dependent on group. Thus, the group difference in learning is exclusively restricted to the slope (a figure of the learning curves is provided in Figure S1).

\section{Learning curves as derived from the mixed modeling approach}

Black crosses denote fit values from the mixed model, as outlined in the results section. Red lines indicate mean fit values, and dashed lines indicate the $99 \%$ confidence intervals of the means. As can be seen, variance in learning increases as learning proceeds. Apparent differences between the intercepts are due to different proportions of men and women, and a different use of tranquilizers across the groups. Moreover, healthy control (HC) achieves an overall maximum number of items recalled during trial five, suggesting a reduced rate of learning in both the at-risk mental state (ARMS) and the first-episode psychosis (FE) patients.

\section{Construction of the serial position curve and determination of the recency effect}

Item-wise recall probabilities were computed by assigning 1.0 to a successful recall and 0.0 otherwise. By summing up the successes of recalling a particular word across trials and dividing it by 5 (total number of trials), probabilities were derived that ranged from 0 (no recall across the trials) to 1 (permanent recall). In order to calculate the primacy and recency (rec) effect, the complete 16-items word list was subdivided into groups of four adjacent list items. The aggregation of the first quarter yielded the primacy effect and the aggregation of the last quarter provided the rec effect, respectively. Therefore, each individual's serial position curve was represented by a series of four averaged recall probabilities (primacy, middle 1, middle 2, and rec).

\section{Variable transformations according to the multivariate analysis of variance and Structural Equation Modeling}

As to satisfy residual normality and heteroscedasticity positional recall probabilities $p_{R}$ were arcsine transformed as follows (and additionally $z$-transformed):

$$
\mathrm{p}_{\mathrm{R}}{ }^{\prime}=\operatorname{asin}\left(\mathrm{p}_{\mathrm{R}}\right) \times \pi / 2+\mathrm{p}_{\mathrm{R}}
$$

For the structural equation modeling, additional variables were transformed in order to meet the above distribution requirements:

1. A working memory composite score was created by calculating the mean from $z$-transformed omission errors and logarithmized reaction times.

2. Cannabis use was dichotomized by collapsing the classes "daily" and "weekly" in "frequent use" and the classes "monthly," "less then monthly," and "no" in "less frequent use" (Table 2).

3. rec recall probabilities were corrected for gender by extracting the residuals from regressing gender on the recall probabilities and dividing them by the standard error of the regression model. This resulted in $z$-values corrected for gender.

4. Slope was corrected for cannabis use (see issue 2) in the same way as issue 3 .

5. A somewhat different approach was used to preprocess the concept failures from the Wisconsin Card Sorting Test. Due to a large floor effect, the distribution of the concept failures was highly skewed. Therefore, the variable was dichotomized according to subjects who scored more than one and others who scored less.

6. The semantic cluster ratio was simply $z$-transformed by centering the values on the mean and dividing them by the standard deviation. 
Table SI Diagnostic criteria for ARMS and FE patients (Yung et al., 2003)'

\begin{tabular}{|c|c|c|}
\hline Patients & $\begin{array}{l}\text { Inclusion and } \\
\text { acute psychosis } \\
\text { criteria }\end{array}$ & Definition \\
\hline$\overline{F E}$ & $\mathbf{A}$ & $\begin{array}{l}\text { Acute psychosis: psychotic symptoms above the transition cut-off (BPRS scales: any ratings of hallucination at } 4 \text { or } \\
\text { above, unusual thought content at } 5 \text { or above, suspiciousness at } 5 \text { or above, conceptual disorganization at } 5 \text { or above) } \\
\text { at least several times a week and persistent for more than a week. }\end{array}$ \\
\hline \multirow[t]{4}{*}{ ARMS } & Al & $\begin{array}{l}\text { "Attenuated" psychotic symptoms: psychotic symptoms below the transition cut-off (BPRS scales: ratings of hallucinations } \\
\text { at } 2-3 \text {, unusual thought content } 3-4 \text {, or suspiciousness } 3-4 \text { ) at least several times per week persisting for }>\mid \text { week. }\end{array}$ \\
\hline & $\mathbf{A 2}$ & $\begin{array}{l}\text { BLIPS: psychotic symptoms over the transition cut-off (BPRS scales: hallucinations } \geq 4 \text {, unusual thought content } \geq 5 \text {, } \\
\text { suspiciousness } \geq 5 \text {, conceptual disorganization } \geq 5 \text { ), but each symptom lasting }<1 \text { week before resolving spontaneously. }\end{array}$ \\
\hline & B & $\begin{array}{l}\text { Genetic risk category: first- or second-degree relative with psychotic disorder and at least two further risk factors } \\
\text { according to the screening instrument. }\end{array}$ \\
\hline & C & Precondition for all categories: criteria of transition to psychosis remain unfulfilled. \\
\hline
\end{tabular}

Notes: ARMS patients fulfill either AI or A2. If none of them is appropriate, criterion B must apply. Criterion C is mandatory for all subgroups of ARMS patients. Abbreviations: ARMS, at-risk mental state for psychosis; BLIPS, Brief Limited Intermittent Psychotic Symptoms; BPRS, Brief Psychiatric Rating Scale; FE, first-episode psychosis.

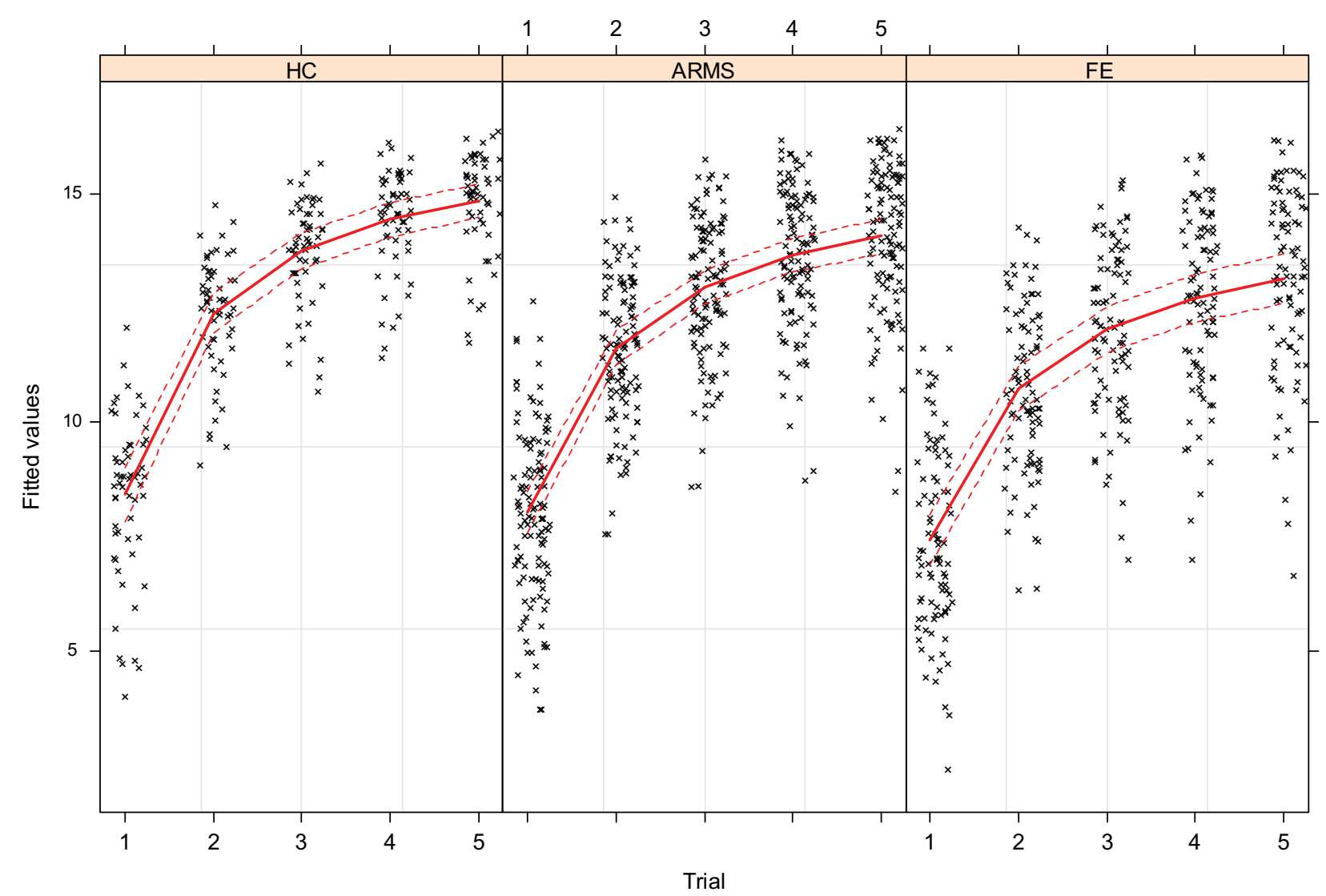

Figure SI Learning curves of the CVLT word list for HC, ARMS, and FE as a function of trials I to 5.

Abbreviations: ARMS, at-risk mental state for psychosis; CVLT, California Verbal Learning Test; FE, first-episode psychosis; HC, healthy control.

\section{Variable imputation}

Initially a small proportion of $0.6 \%$ data were missing, most frequently affecting cannabis use. In total, seven variables were affected by missing values (working memory omission errors and reaction time, Wisconsin Card Sorting Test concept failure, Brief Psychiatric Rating Scale global score, Scale for the Assessment of Negative Symptoms summary score, recall from trial 6 of the California Verbal Learning Test, and cannabis use).
The imputation of the missing values was achieved by using the Random Forest rfImpute procedure from the $\mathrm{R}$ package randomForest 4.6-12.

The algorithm starts by imputing "NA"s using "na. roughfix" (which is a starter function, likewise provided by the randomForest package). Then, the "randomForest" procedure is called with the completed data. The proximity matrix from the randomForest is used to update the imputation of the "NA"s. For continuous predictors, the imputed value is 
the weighted average of the nonmissing observations, where the weights are the proximities. For categorical predictors, the imputed value is the category with the largest average proximity. This process is iterated multiple times.

\section{Reference}

1. Yung AR, Phillips LJ, Yuen HP, et al. Psychosis prediction: 12-month follow up of a high-risk ("prodromal") group. Schizophr Res. 2003;60(1): 21-32.

\section{Publish your work in this journal}

Psychology Research and Behavior Management is an international, peerreviewed, open access journal focusing on the science of psychology and its application in behavior management to develop improved outcomes in the clinical, educational, sports and business arenas. Specific topics covered in the journal include: Neuroscience, memory and decision making; Behavior modification and management; Clinical applications; Business and sports performance management; Social and developmental studies; Animal studies. The manuscript management system is completely online and includes a very quick and fair peer-review system, which is all easy to use. Visit http://www. dovepress.com/testimonials.php to read real quotes from published authors.

Submit your manuscript here: https://www.dovepress.com/psychology-research-and-behavior-management-journal 